\title{
A Comparison of Two Data-Driven Models to Predict Hypolimnetic Dissolved Oxygen Concentration: A Case Study of the Seymareh Reservoir in Iran
}

\author{
A. K. Nokhandan ${ }^{1}$, E. Snieder ${ }^{1}$, U. T. Khan ${ }^{1 *}$, A. ElDyasti ${ }^{1}$, Z. Ghaemi ${ }^{2}$, and M. Bagheri ${ }^{2}$ \\ ${ }^{1}$ Department of Civil Engineering, Lassonde School of Engineering, York University, Toronto, Ontario M3J1P3, Canada \\ ${ }^{2}$ Mahab Ghodss Consulting Engineering Company, Tehran 1918783414, Iran
}

Received 8 Augest 2019; revised 13 September 2019; accepted 5 October 2019; published online 5 November 2019

\begin{abstract}
Dissolved oxygen concentration (DO) is a crucial factor in maintaining aquatic ecosystem health. In this research, two data-driven modelling (DDM) techniques, multiple linear regression (MLR) and artificial neural networks (ANN), were developed, implemented and compared to predict the DO in the hypolimnetic layer of Seymareh Reservoir in Iran. Low DO in this Reservoir lead to a fish kill event and thus, this reservoir is of interest to water managers in the region. Water quality monitoring data from the Reservoir and an upstream river were used for training the models. In addition, two input variable selection methods, linear correlation analysis and combined neural pathway strength analysis (CNPSA, a nonlinear variable selection method) were developed and compared to determine the most significant inputs to predict hypolimnetic DO. A systematic method to select the optimum architecture of the network is proposed and tested. While these two approaches have been investigated previously, this research focuses on creating a systematic approach to combining two sources of uncertainty of DDM models. Additionally, the performance of CNPSA has not been compared to linear variable selection techniques. This research demonstrates the importance of using systematic input selection and network design for improved DO prediction in a large Reservoir. The performance of the models was quantified using the Nash-Sutcliffe efficiency and root mean squared error, which demonstrated that the ANN approach had better performance compared to the MLR model. The approach demonstrates that by using a systematic input variable selection approach combined with an optimised network architecture, a high performance of DO prediction can be achieved using easily measured upstream input data.
\end{abstract}

Keywords: water quality, artificial neural networks, multiple linear regression, dissolved oxygen, input variable selection

\section{Introduction}

Dissolved oxygen concentration (DO) is one of the most crucial water quality indicators and it can influence a number of processes in lakes and reservoirs, such as nitrification and algal growth. Low DO, especially in the deeper layers of reservoirs (i.e., the hypolimnion layer), can lead to the release of phosphorus, nitrogen, methyl-mercury, hydrogen sulphide, iron, and manganese from the bed sediment due to a number of anaerobic biogeochemical mechanisms (Bierlein et al., 2017). The release of these substances into waterbodies can enhance the trophic state of the waterbody leading to additional oxygen demand. These interactions pose a major risk to the aquatic ecosystem. In addition, the absence or low levels of DO can cause fish kills and odour in potable water. For instance, several studies, e.g., Burkholder et al. (1999), Lopez et al. (2008), and Small et al. (2014), have investigated the reasons of fish kill events in reservoirs, and in fact, hypoxia is one of the leading causes. More recently, major fish kill events on the coast of Nova Scotia, Canada (in 2016) and in the Pampanga River in

* Corresponding author. Tel.: +1 4167362100 ext. 55890

E-mail address: usman.khan@lassonde.yorku.ca (U. T. Khan).

ISSN: 2663-6859 print/2663-6867 online

(C) 2019 ISEIS All rights reserved. doi:10.3808/jeil.201900017.
Masanto, Philippines (in 2017) have both been attributed to hypoxic conditions.

Given the consequences of low DO on aquatic ecosystems, it is not surprising that numerous studies on lakes and reservoirs water quality have considered DO as one the most crucial indicator of overall water quality. Accurate predictions of both short and long-term DO are vital for water resources managers and an important decision-making factor (Stefan and Fang, 1994; Hamilton and Schladow, 1997; Cooke, 2005). Several concepts and models have been used to predict DO in lakes and reservoirs such as 1, 2 or 3-D physically-based models, as well as data-driven models (including simple linear regression, and neural networks). The use of these models to predict DO is summarised below.

The simplest physically-based modelling approach is using a one-dimensional model such as MIKE-11 and QUAL2K. Rucinski et al. (2010) developed a one-dimensional DO model in the central basin of Lake Erie (USA-Canada). In this study, the model considered simplified biological processes in the Lake in order to focus on the effects of vertical stratification and mixing dynamics. This model computed daily vertical profiles of temperature and DO for the period $1987 \sim 2005$. Model calibration resulted in good agreement with observations of the thermal structure and oxygen concentrations throughout the 
period of study. The model was calibrated using seasonal oxygen concentrations by adjusting the water column oxygen demand, while other factors such as pollutant concentration were used to determine DO. However, note that one-dimensional models are more appropriate for rivers rather than reservoirs because of their weaknesses in simulating the water quality along the length of the reservoirs.

Consequently, two or three-dimensional physically-based models have been developed for modelling DO in dams and reservoirs, in part to overcome the limitations of the one-dimensional approach. A number of relevant studies have been done in this field. Brito et al. (2018) simulated the water quality (including DO) of Enxoe Reservoir (in Portugal) using a twodimensional modelling tool (CE-QUAL-W2) with inputs from a watershed model (SWAT). They modelled the trophic level of the reservoir and evaluated the efficiency of different mitigation plans by the modelling tools. The CE-QUAL-W2 modelling tool considers property sources and sinks, interactions between temperature, nutrients, algae, DO, organic matter, and sediments for its calculations. In terms of calibration and validation, up to 50 different parameters were calibrated for the Enxoe Reservoir; this demonstrates one major drawback of physical-based models, namely, the complexity and difficulty in calibration when a large number of parameters are considered. The modelling results showed that the model tended to underestimate DO near the bottom during the summer months.

Additionally, Trolle et al. (2014) undertook a three-dimensional water quality model to examine the impacts of agricultural intensification in the watershed of Lake Benmore (in New Zealand). A large amount of information was required as inputs of the modelling, including daily inflow and outflow, water temperature, DO, and nutrient concentrations in inflows, and hourly or daily meteorological data (air temperature, shortwave radiation, relative humidity, wind speed, wind direction, and rainfall). Similarly, Bocaniov et al. (2017) examined the temporal and spatial hypoxia development (especially in the hypolimnetic layer) and its response to the nutrient load in Lake Erie (USA-Canada) using a three-dimensional lake model. The modelling tool was aimed to simulate water and sediment chemical and biological processes. To summarise, given the complex physical systems that govern DO in reservoirs and lakes, two- and three-dimensional physically-based models are necessary. However, these types of models are increasingly complex (to account for interactive effects between air, water and sediment), hard to calibrate (which may introduce additional uncertainties in the model), and require a large amount of site specific data (which are not easily available). Thus, an alternative option is to use data-driven models. In these models, a mathematical structure is developed that is capable of providing the linear or non-linear relationship between inputs and outputs. The data driven approach concentrates on forming a system that can develop the answer based on what has been seen before.

Data-driven models (DDMs) and machine learning methods have recently gained wide interest in modelling DO, in part due to reduce the uncertainty present in physically-based models. These techniques try to predict the water quality parameters (e.g., DO) based on the temporal and spatial relationship of observed water quality dataset without the explicit knowledge of the underlying physical system (Solomatine and Ostfeld, 2008). DDM includes different categories of statistical and artificial intelligent models such as artificial neural networks (ANNs), fuzzy-based systems, and multiple linear regression (MLR) (Khadr and Elshemy, 2017).

Regression models have been used in a wide array of applications including econometrics, finance, sociology, hydrology, biology, psychology, pharmacology, and engineering studies. This method develops relationships between the dependent variable (e.g., a water quality parameter like DO) and selected independent variables by fitting a linear equation to the observed data set. However, ANNs are an alternative type of DDM; they are well suited for many environmental or hydrological applications because of their informative processing characteristics, such as nonlinearity, parallelism, noise tolerance, and learning and generalization capabilities (Liu and Chung, 2014). In recent years, several research studies have been conducted on water quality simulation using these techniques. Basant et al. (2010) simulated DO and BOD 5 in the Gomti River (India) by the application of partial least squares regression and feed forward back propagation ANN using a set of independent measured variables (specifically $\mathrm{pH}$, TSS, alkalinity, hardness, $\mathrm{Cl}^{-}, \mathrm{PO}_{4}{ }^{3-}, \mathrm{K}^{+}, \mathrm{Na}^{+}, \mathrm{NH}_{4}{ }^{+}-\mathrm{N}, \mathrm{NO}_{3}^{-}-\mathrm{N}$ and $\mathrm{COD})$. The performance of these models were assessed by different criteria such as root mean squared error (RMSE), the standard error of prediction (SEP), the coefficient of determination $(\mathrm{R})$, the Nash-Sutcliffe efficiency $\left(R^{2}\right)$, and the accuracy factor (Af). The results illustrated that the nonlinear model (ANN) performed better than the linear one.

He et al. (2011) analysed different factors that contribute to DO levels in a wastewater-impacted river in Calgary, Canada using statistical data analysis and modelling. The results illustrated that both climatic conditions (reflected in water temperature) and hydrometric conditions (flow) were major factors that influence DO in the Bow River. In their study, the nonlinear approach using multiple-layer perceptron neural network, showed a better performance compared to the MLR approach. Khan and Valeo $(2015,2016$, and 2017) proposed new methods for DO prediction in the same river in Calgary using both MLR and ANN techniques by accounting for uncertainty by using fuzzy numbers. The methods further demonstrated the ability of DDMs to predict DO in complex, urbanised watersheds, and had the ability to predict the risk of low DO events as well.

Heddam and Kisi (2018) implemented three types of artificial intelligence techniques, least square support vector machine, multivariate adaptive regression splines, and M5 Model Tree in order to predict the concentration of DO in different stations in different rivers in the USA. Three indices including $\mathrm{R}, \mathrm{RMSE}$ and mean absolute error (MAE) were used to assess and evaluate the model performance. The results of this study also showed that these DDM techniques have the capacity to predict DO, although the best model differs for one station to another, and are also a function of which data is available.

These studies highlight the utility and need for DDMs for 
water quality prediction, particularly, for DO in complex systems where physically-based models may not be appropriate. However, in most of these studies, an ad hoc or trial and error approach is used design the DDMs. In particular, a consistent method to select the inputs (i.e. the independent variables) is needed to develop the best performing models. Additionally, the structure of the DDMs (i.e. the number of input parameters or model coefficients) are not explored.

Input variable selection (IVS) is a key factor in DDM development. Most studies use linear correlation analysis to determine the best input variable. While this can be a robust way to select inputs for MLR, however, it is not a suitable approach for ANN or other non-linear models. The major disadvantage is that it is unable to account any nonlinear dependence that may exist between the input and the output datasets, and may possibly result in the elimination of important input parameters that have a nonlinear relationship with the output variable. There are several methods to conduct IVS for ANNs. He et al. (2011) investigated the contribution of different factors in the level of daily minimum DO using MLR and ANN. For MLR, the candidate input variables were determined according to the partial correlation between input and output, while for the ANN model performance was generally improved by adding more independent variables as compared to MLR. Valeo and $\mathrm{He}$ (2009) used an ANN and fuzzy logic based method to obtain quantile estimates for rainfall prediction. This study compared conventional parametric methods and found that conventional models require the selection of the underlying distribution of the process while ANNs do not require a priori identification of a suitable distribution. The results showed the better performances of ANN modelling techniques. More recently, Khan et al. (2018) tested the combined neural pathway strength analysis (CNPSA) method for input selection process for urban flood prediction. This process measured the relative strength of an input and the output of an ANN to determine those inputs that have the highest impact in predicting the output. These are two of several methods that can be used for IVS for ANN or other DDM; a detailed review can be found in Quilty et al. (2016). Most DDM approaches for modelling DO or other hydrological variables do not include a systematic IVS approach. However, recent studies have understood and demonstrated the importance of this crucial step. In addition to IVS, the structure of DDMs needs to be designed accurately. In fact, an inappropriate structure of a DDM may cause the model to overfit the training data and reduce the ability of the model to generalize.

With respect to all the gaps and limitations of the previous studies, in this research, the application of two DDM methods (namely, MLR and ANN) with a systematic IVS approach and an accurate design of the model structure is explored. Data from the Seymareh Reservoir in western Iran is used to demonstrate the proposed modelling approach. The Reservoir was primarily built for hydropower generation and as a flood control facility. In 2012, the low DO in the water caused a fish kill event in the reservoir, which was considered an environmental disaster. Thus, local water resources managers were interested in developing an accurate method to estimate DO to better understand the conditions leading to low DO, to predict future low
DO events, and to evaluate various mitigation plans to help improved DO levels. Therefore, the main objective of this study is to develop two data-driven methods (MLR and ANN) to simulate the DO level in the hypolimnetic layer of the Seymareh Reservoir in Iran. Two IVS methods were explored and compared to design the data-driven models: linear correlation method is used to select the inputs for the MLR model, and the CNPSA IVS method is used to select the inputs for the ANN model. The proposed models will allow reservoir and dam operators and water resource managers to predict DO in the deeper layers of reservoirs using upstream river water quality parameters. The significance of using upstream river water quality data is that it allows the prediction of DO in the deeper hypolimnetic layer of the Reservoir without needing to collect data from this difficult to access and measure location. While this research shows results from one study area, the proposed approach is generalizable to other regions. The innovation of this research is a systematic IVS and network architectural optimisation technique of ANN models. This analysis can help better understand the physio-chemical mechanisms that affect hypolimnetic DO in reservoirs, leading to more accurate predictions of DO, and thus preventing detrimental impacts to water bodies.

\section{Methods}

\subsection{Study Area}

The Seymareh Reservoir (Figure 1) has a total watershed area of $27,000 \mathrm{~km}^{2}$ and an effective storage about $2500 \times 106$ $\mathrm{m}^{3}$ at normal elevation. In 2012, low DO resulted in a fish kill event in the Reservoir. The main reason for the low DO in the upper layer of the reservoir was the considerable anaerobic zone in the hypolimnion (deepest) layer that affected the entire waterbody, which in turn lowered the DO.

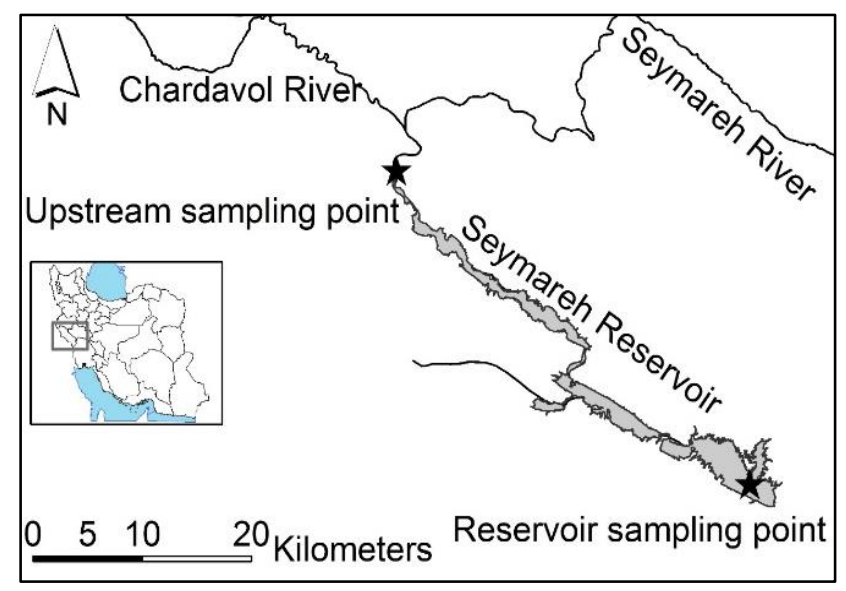

Figure 1. The seymareh reservoir and upstream watershed (the location of the reservoir within the larger area is shown in the inset).

Due to this event, a water quality monitoring program was initiated in 2013 for a one-year period as a part of a water quality management project under the supervision of Ministry 
Table 1. A Statistical Summary of the Water Quality Variables in the Seymareh Reservoir and Upstream Inflow ( $n=34$ samples)

\begin{tabular}{lllllll}
\hline & Reservoir & & \multicolumn{3}{l}{ Inflow } \\
\hline Variable & Min & Mean & Max & Min & Mean & Max \\
Water temperature $\left({ }^{\circ} \mathrm{C}\right)$ & 11 & 18.3 & 28.5 & 7.2 & 18.4 & 29 \\
$\mathrm{pH}$ & 6.9 & 7.6 & 8.7 & 7.5 & 7.8 & 8.3 \\
Electrical Conductivity & 532 & 784 & 1270 & 491 & 761 & 1180 \\
$(\mu \mathrm{mohs} / \mathrm{cm})$ & 8.1 & 20.5 & 39.3 & 17.8 & 22.2 & 28.5 \\
$\mathrm{COD}(\mathrm{mg} / \mathrm{L})$ & 0.7 & 6.6 & 11.7 & 7.3 & 9.9 & 13.6 \\
DO $(\mathrm{mg} / \mathrm{L})$ & 0.9 & 2.3 & 4.6 & 1 & 2.95 & 5.1 \\
Total Nitrogen $(\mathrm{mg} / \mathrm{L})$ & 0.07 & 0.2 & 0.46 & 0.11 & 0.29 & 0.51 \\
Total Phosphorus $(\mathrm{mg} / \mathrm{L})$ & & &
\end{tabular}

of Energy in Iran by Mahab Ghodss' laboratory. There were two water quality sampling stations that collected data three times per month (except in December when only one sample was collected, resulting in 34 samples for the year). One station was located on at the upstream river just before the entrance of the Reservoir and the second station was located in the main Reservoir itself near the dam body (Figure 1). The distance between these two stations was about $40 \mathrm{~km}$. In addition to the water quality parameters (see Table 1 for a complete list of parameters); other parameters related to the Reservoir such as wind speed (W), Secchi disk (Sd) and water depth (D) were also measured. A Horiba Portable U-10 Water Quality sampler was used to measure in-situ Electric Conductivity (EC), water temperature (T), $\mathrm{pH}$, and Dissolved Oxygen (DO). Manual (grab) samples were collected from the upstream and downstream location to measure 5-day Biochemical Oxygen Demand $\left(\mathrm{BOD}_{5}\right)$ and Carbonaceous Oxygen Demand (COD), Total Phosphorus (TP), and Total Nitrogen (TN) using Standard Methods. To collect samples from the hypolimnetic layer, a custom-made vertical point water sampler was used. All manual samples were transferred to an analytical water quality laboratory in Tehran within 24 hours for analysis.

Both the water quality data and the additional physical parameters were considered as candidate inputs for the two DDMs in this research. Table 1 summarises the descriptive statistics of this sampling program. On average, the table summarises the spatial distribution of the parameters, highlighting that the concentrations of TP, TN, DO, COD, and $\mathrm{pH}$ are lower within the reservoir as compared to the upstream - in particular DO, which reduced from $9.9 \mathrm{mg} / \mathrm{L}$ to $6.6 \mathrm{mg} / \mathrm{L}$. On the other hand, EC had a slightly higher value in the reservoir compared to the upstream location, whereas the water temperature was essentially constant. From a temporal perspective, the concentrations of water quality were generally higher (with the exception of DO) in the summer months (which is the dry period in the study region) due to lower inflows and constant point source pollution from upstream municipal and industrial wastewater sources. Winter concentrations were lower due to higher inflows into the reservoir. For the DO, the minimum, mean, and maximum values for the Reservoir are $0.7,6.6$, and $11.7 \mathrm{mg} / \mathrm{L}$, respectively. Note that the minimum amount is well-below the water quality guidelines for the protection of aquatic habitat which is $5 \mathrm{mg} / \mathrm{L}$ based on the Iranian Department of Environment Standards.
All the data presented in Table 1 was used to predict DO using either the MLR or ANN approach, following the systematic IVS approaches described below. Previous studies have identified that a key step in selecting inputs for these types of model is to normalise the inputs (Khan et al., 2018, Saghi et al., 2015). Therefore, the inputs and target amounts were normalised using the following equation (Equation (1)):

$X_{n}=\left(Y_{\max }-Y_{\min }\right)\left(\frac{X-X_{\min }}{X_{\max }-X_{\min }}\right)-Y_{\text {min }}$

where $X$ is the data value, $X_{n}$ is the normalized data value, $X_{\max }$ and $X_{\min }$ are the maximum and minimum values of all data and $Y_{\max }$ and $Y_{\min }$ are maximum and minimum values in the range of $(0,1)$.

\subsection{Model Performance Metrics}

For this research, two common model performance metrics were used to measure and compare model performance: the $R M S E$ and the Nash-Sutcliffe efficiency $\left(R^{2}\right)$. These metrics were calculated using the following equations:

$$
\begin{aligned}
& R M S E=\sqrt{\frac{1}{N} \sum\left[C_{p}-C_{m}\right]^{2}} \\
& R^{2}=1-\frac{\sum\left(C_{p}-C_{m}\right)^{2}}{\sum\left(C_{m}-\underline{C_{m}}\right)^{2}}
\end{aligned}
$$

where $N$ is the total number of data points, $C_{p}$ is a simulated water quality variable, $C_{m}$ is a measured water quality variable, and $C_{m}$ denote the average of measured water quality variables.

\subsection{Multiple Linear Regression}

The general form of multiple linear regression models is:

$y=\beta_{0}+\beta_{1} x_{1}+\beta_{2} x_{2}+\ldots+\beta_{k} x_{k}+r$

where $\beta_{0}$ is the intercept, $\beta_{1}$ is the parameter associated with $x_{1}$, $\beta_{2}$ is the parameter associated with $x_{2}$ and the variable $r$ is the residual. The MLR model is trained by minimizing the sum of 
the squares of the residuals between the predicted and the observed data (Kovdienko et al., 2010; Ferraro and Giordani, 2012). Of the 34 samples, $80 \%$ (27 samples) and $20 \%$ (7 samples) of the input data were used for training and testing (i.e. independent dataset) the regression model, respectively. The selection of an appropriate set of input variables for DDMs is a very important step and has been identified as the major source of uncertainty in DDM implementation (Khan and Valeo, 2017). It is difficult to determine how many and which input variables (from the full dataset shown in Table 1) should be used in the model. A common approach to select the appropriate inputs for MLR is to determine the correlation coefficient $(R)$ between the candidate input variables $(x)$ and DO $(y)$ in the hypolimnion layer based (using Equation (5)). The higher the value of $R$ the better candidate that particular variable is as an input:

$$
R=\frac{n\left(\sum x y\right)-\left(\sum x\right)\left(\sum y\right)}{\sqrt{\left[n \sum x^{2}-\left(\sum x\right)^{2}\right]\left[n \sum y^{2}-\left(\sum y\right)^{2}\right]}}
$$

where $x$ and $y$ represent the input variables and hypolimnion DO respectively. In addition, there are several assumptions related to the MLR models that need to be met for the model to be applicable. Typically, after a regression analysis has been conducted, an analysis of residuals must be conducted to ensure that these assumptions of the model have not been violated. In this study, it has assumed that the measurement of DO was error free. The four principal assumptions of linear regression are (Khan and Valeo, 2016):

(i) the mean of residuals $r$ is zero, $E(r)=0$;

(ii) the residuals, $r$, have a constant variance with respect to time, i.e. the data is homoscedastic $\operatorname{Var}(r)=\sigma^{2}$;

(iii) the residuals, $r$, are independent or uncorrelated; and

(iv) the residuals, $r$, are normally distributed, $r \sim N\left(0, \sigma^{2}\right)$.

For the third assumption, the Durbin-Watson statistic test (Durbin and Watson, 1951) can be used to determine whether or not the residuals are independent. In this test, the test statistic, $d$ (see Equation (6)) is calculated using the residual $r$. The value of $d$ lies between 0 and 4 ; if $d \geq 2$, it indicates no autocorrelation, and if $d$ is substantially less than 2 , there is evidence of positive serial correlation. In regression, this can imply an underestimation of the level of statistical significance:

$d=\frac{\sum_{t=2}^{T}\left(r_{t}-r_{t-1}\right)^{2}}{\sum_{t=1}^{T} r_{t}^{2}}$

\subsection{Artificial Neural Network and CNPSA}

In addition to the MLR approach, a multilayer perception, feed-forward ANN model was developed to predict the hypolimnetic DO in the Seymareh Reservoir. In this case, 70\% (24 samples), $15 \%$ (5 samples) and 15\% (5 samples) of the input data were used for the training, validating and testing phases, respectively. Feed forward neural networks propagate data from input to output and they are the most popular and widely used ANN models. These models are often made of one input layer, one or more hidden layers, and an output layer. The general formula for the data transfer from the input layer to the output layer is defined by Equation (7):

$Y_{i}=\sum_{j=1}^{Z} W_{i j} X_{j}+b$

where $Z$ is the number of neurons in the last layer, $i$ is the number of outputs, $X_{j}(j=1, Z)$ are the input data from neurons in the last layer for i-th output, $W_{i j}(j=1: Z)$ are the weights of $X_{j}$ neurons and $b$ is the bias. The main differences between the various types of ANNs include: the number of hidden layer number; the number of neurons in each layer; the activation function used in each neuron and the training methods used (e.g., the commonly used back-propagation Levenberg-Marquadt method) (Saghi et al., 2015).

As mentioned above, different input combinations can change the performance of the ANN models. Therefore, an IVS method should be considered to identify the most effective input parameters and the best combinations of inputs to predict DO in the Reservoir. One approach to do this is to use the relatively recently developed Combined Neural Pathway Strength Analysis (CNPSA) method (Duncan, 2014; Khan et al., 2018). The CNPSA can calculate the most significant input parameters by calculating the relative strength of a particular pathway from each input to the output within the ANN. The strength is calculated using the magnitude of the weights (i.e., the tuning parameters) of the ANN as shown in Equation (8):

$W_{I O}=W_{I H} \cdot W_{H O}$

where $W_{l H}$ is representing the weights between the inputs, and hidden layer, and also $W_{H O}$ are the weights between the hidden layer and output later. The greater the value of $W_{I O}$ the better input variable in predicting the output using the ANN. For the modelling of hypolimnetic DO in Seymareh Reservoir, first, the significance of each input is calculated, the $W_{I O}$ are collected and used to calculate the ensemble interquartile range (EQR) for each of the inputs as based on Eqation (9):

$E Q R=\frac{\left(\left|Q_{1}\right|,\left|Q_{3}\right|\right)}{\max \left(\left|Q_{1}\right|,\left|Q_{3}\right|\right)} \operatorname{sign}\left(Q_{1}\right) \operatorname{sign}\left(Q_{3}\right)$

where $Q_{l}$ and $Q_{3}$ are the first and third quartile of the $W_{I O}$.

Determining the optimum number of nodes in the hidden layer and the transfer functions are also an important component of the design and architecture of an ANN model. An excessively large number of nodes (and hence weights and biases) may result in overfitting, while an insufficient number of nodes may not capture the information adequately (e.g. Dawson and Wilby, 2001; Singh et al., 2009; Ranković et al., 2010). Furthermore, the number of neurons in the hidden layer 
can affect the strength of every input and as a result in this research different numbers of neurons in the hidden layer (specifically $3,6,9,12,15$ and 18 neurons) were examined in order to implement the CNPSA. After determining the most effective input variables, the best network architecture for ANN model was obtained based on the $R^{2}$ and RMSE values from the testing dataset.

\subsection{Hypothesis Testing for Model Comparison}

In this research, five hypothesis tests including the t-test (Kalpić et al, 2011), the rank-sum test, Signed-rank test (Wilcoxon, 1945), the KS-test (Newman, 1939), and the F-test (Snedecor and Cochran, 1989) were used to determine whether there was a statistically significant difference between the modeled output and observed DO, as well as to compared the two models. The 5\% significance level was used to determine if the results were significantly different or not. All model development and hypothesis tests were performed using MATLAB (2017a).

\section{Results and Discussion}

\subsection{Multiple Linear Regression Model}

The correlation coefficient was calculated for the DO in the hypolimnetic layer of the reservoir and all input variables (including the water quality parameter in the river, and the physical reservoir characteristics). A summary of these calculations is listed in Table 2 which demonstrate that five most effective input are EC, TP, T, DO, and W (all with an $R^{2}$ value greater than 0.3). Note that the other parameters such as $\mathrm{BOD}_{5}$, $\mathrm{TN}, \mathrm{Sd}$, and D, did not show a sizable correlation with the hypolimnetic DO and were thus, eliminated from further consideration. The low $R^{2}$ values indicate the lack of a linear relationship between any single variable and the DO in the hypolimnetic layer.

Also, with respect to the value of $R^{2}$ between these five important parameters: TP, DO and $\mathrm{W}$ are directly correlated while EC and T are inversely related. For DO and W it is obvious that by increasing the DO at the upstream river and also by having more wind speed, it is expected to correspond to higher levels of DO in the hypolimnion layer. An increase in TP concentration usually happens at the beginning of the wet season with an increase in precipitation. At the same time, the thermal stratification of the reservoir starts to change, and mixing of the water body begins which is an important event for increasing the DO level of the whole water body (including the hypolimnion layer) and as a result, the increase in the hypolimnetic DO and TP occur at the same time. On the other hand, EC usually increases during the dry seasons when contaminant concentration is higher owing to lower water volumes. Also, hypoxic conditions and low DO in the deeper layers of the Reservoir usually occur in the summer. Thus, lower hypolimnetic DO levels are observed when T and EC are both higher.

Following this, several MLR models were constructed to examine the efficiency of different input combination in esti- mating the output. Each model has a different combination of input variables (that were short-listed as candidate inputs using the correlation coefficient). These models were compared using the two model performance criteria $\left(R^{2}\right.$ and RMSE) and are summarised in Table 3.

Table 2. The Correlation Coefficient between Candidate Input Variables and Hypolimnetic DO

\begin{tabular}{ll}
\hline Input variables & $\mathrm{R}$ \\
\hline T & -0.597 \\
DO & 0.566 \\
BOD 5 & -0.0155 \\
EC & -0.902 \\
TN & 0.164 \\
TP & 0.722 \\
D & -0.166 \\
SD & 0.091 \\
W & 0.629 \\
\hline
\end{tabular}

Table 3. The Performance of MLR Modelling with Different Input Combinations

\begin{tabular}{lll}
\hline Input parameters & $R^{2}$ & RMSE \\
\hline T, DO, EC, TP, W & 0.857 & 0.90 \\
T, DO, TP, W & 0.741 & 0.94 \\
T, DO, EC, W & 0.845 & 0.90 \\
T, DO, EC, TP & 0.821 & 0.91 \\
DO, EC, TP, W & 0.845 & 0.90 \\
T, EC, TP, W & 0.857 & 0.89 \\
EC, TP, W & 0.839 & 0.90 \\
EC, TP & 0.814 & 0.91 \\
EC, W & 0.837 & 0.90 \\
EC & 0.814 & 0.91 \\
\hline
\end{tabular}

Based on the results presented in the Table 3, the best performance of the MLR model was achieved when T, EC, TP, and $\mathrm{W}$ were used as the input variables. The regression equation for this model was found as follows:

$$
\text { Hypolimnetic } \begin{aligned}
\widehat{D O}= & 0.2239-0.6632 \widehat{E C}+0.2594 \widehat{T P} \\
& +0.5125 \widehat{W}+0.4160 \widehat{T}
\end{aligned}
$$

Figure 5a compares the observed DO and the predicted DO using this MLR model. The $R^{2}$ value for the entire dataset was 0.857 , while the RMSE was $0.9 \mathrm{mg} / \mathrm{L}$ which is quite low (less than 15\%) comparing the average amount of DO in the reservoir $(6.6 \mathrm{mg} / \mathrm{L})$ and shows a good performance of modelling procedure. The reason for having a direct relationship between $\mathrm{T}$ and Hypolimnetic DO in this equation might be the correlation between $T$ and the other independent variables which is one the weaknesses of using an MLR approach.

In order to use the proposed MLR model, it is necessary to test and verify that the proposed equation (Equation (10)) satisfies the linear regression assumptions. The results of checking the assumptions are presented in Figure 3. The residuals were confirmed to follow the normal distribution 

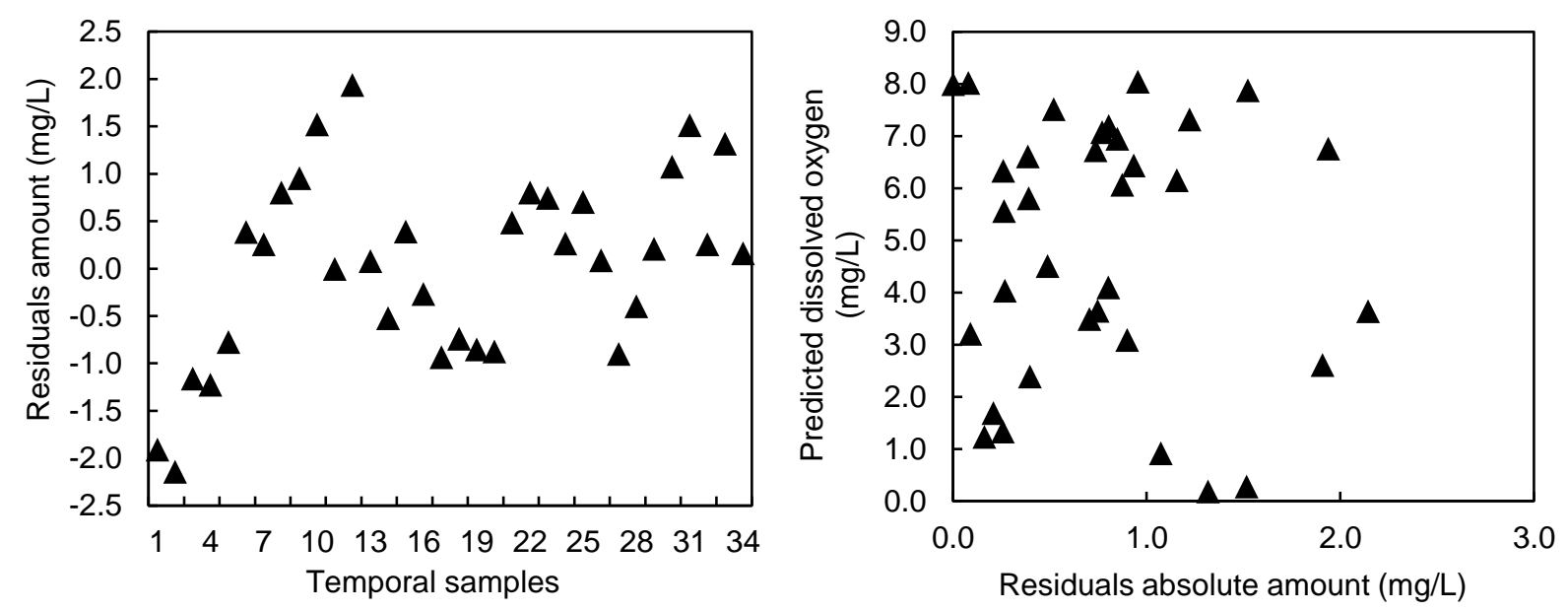

Figure 2. Checking MLR assumptions of (a) correlation of residuals and (b) homoscedasticity.

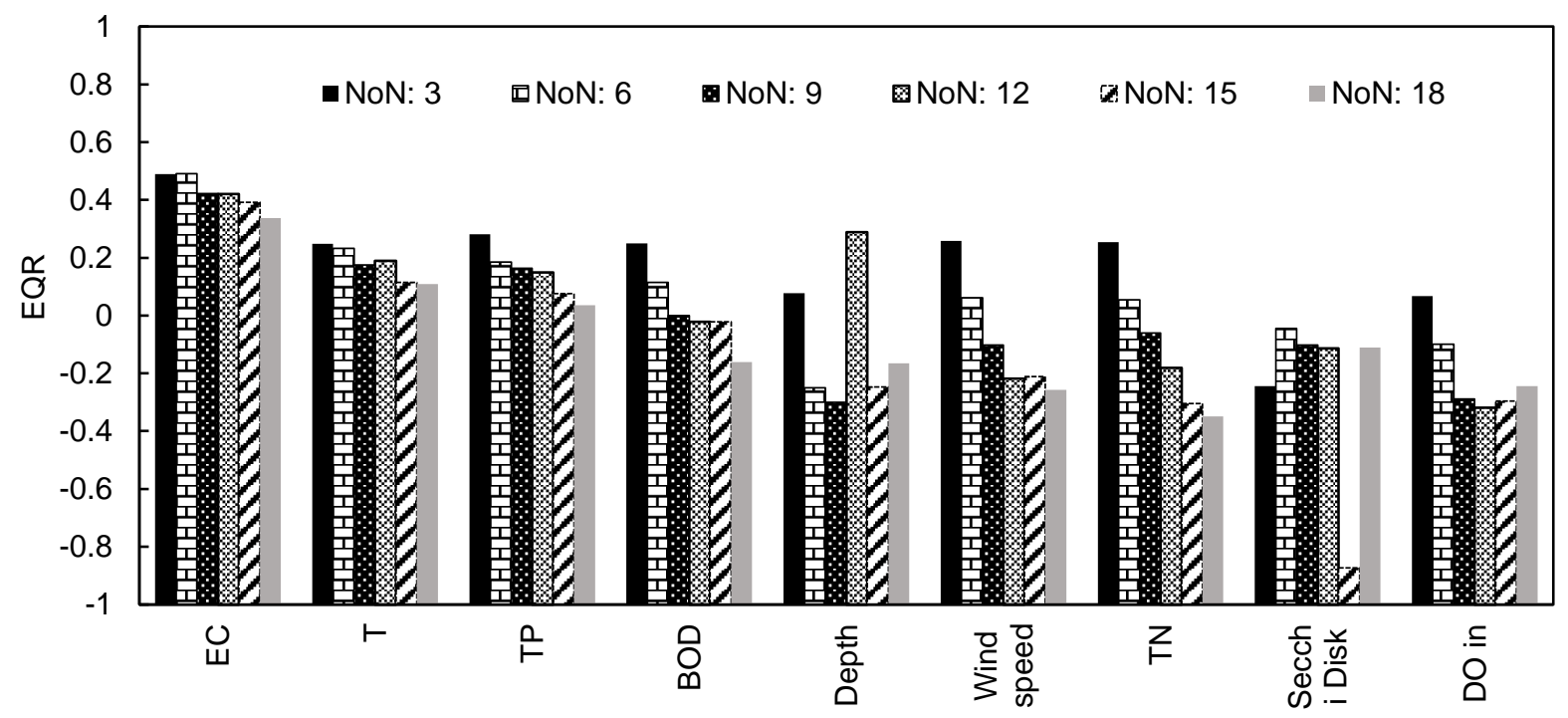

Figure 3. The EQR values for different input parameters.

using the Jarque-Bera test. The autocorrelation assumption (illustrated in Figure 2a), was tested using the Durbin-Watson test statistic $d$ which was equal to 0.8 , suggesting that the residuals are autocorrelated. The assumption of constant variance (homoscedasticity) was not violated (as shown in Figure 2b). Finally, the mean of residuals was calculated to be equal to zero. Therefore, regarding the violation of one of the assumption which implies an underestimation of the level of statistical significance and existence of a correlation between the input variables hence, implying that the MLR estimators are not unbiased.

\subsection{Artificial Neural Network (ANN) Model}

Similar to the MLR approach, as a first step for the ANN model, CNPSA was used for IVS to identify the most effective input combination to predict the hypolimnetic DO model.
Figure 3 illustrates the results of CNPSA for different inputs under several different Number of Neurons (NoN, which were varied from 3 to 18). Based on this analysis, the most important input parameters were identified as EC, T and TP, since these inputs have a positive EQR value for all NoN cases considered.

Thus, these three variables were selected as the final inputs for the ANN model. Note that this selection is similar to the IVS selection for the MLR (using $R$ ) model but excludes wind speed (W). Note that $\mathrm{W}$ had negative EQR for the ANN models with a NoN of 9 and higher.

Following the CNPSA and EQR analyses for IVS the performance of the model for each NoN configuration was examined, and these results are illustrated in Figure 4. The figure shows that generally for each configuration the values of the two metrics, $R^{2}$ and RMSE, suggested good performance for each model. No obvious trends are apparent with decreasing 


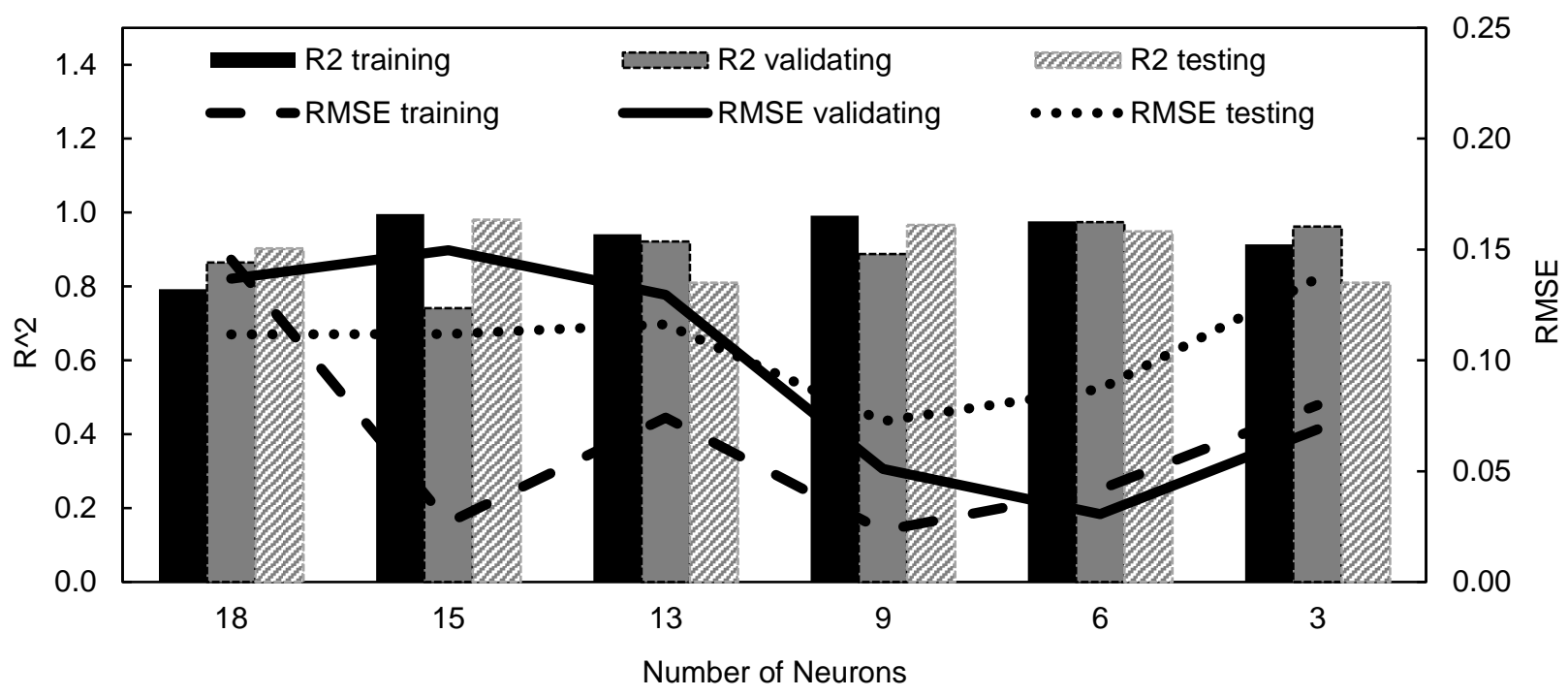

Figure 4. The performances of the model in each phase (training, validating and testing) under different numbers of neurons in the hidden layer.
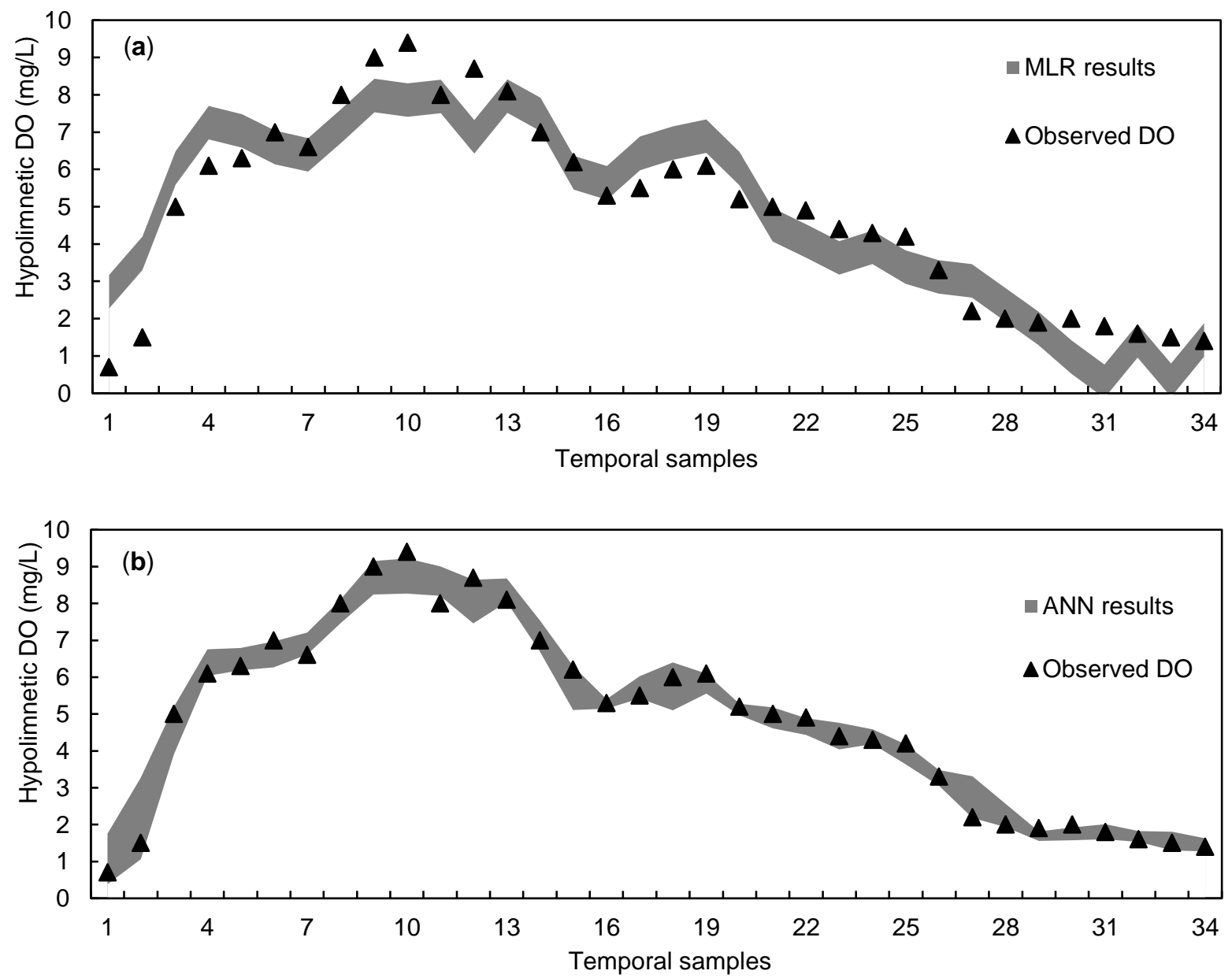

Figure 5. Predicted and measured hypolimnetic DO in the Seymareh Reservoir under different modelling methods (a) MLR and (b) ANN. 
NoN, nor in comparing the testing dataset values to the training and validation datasets. Considering the high values of $R^{2}$ and lower values of RMSE, the model with six NoN was selected as the final architecture and ANN configuration for this research.

To confirm the effectiveness of the CNPSA and EQR approach, the six NoN ANN model was retrained using different combinations of inputs from the short-listed candidates (EC, $\mathrm{TP}$, and $\mathrm{T}$ ) are the results of this are summarised in Table 4 . The data in the table shows that the best performing model is clearly the model that uses all three inputs identified by the CNPSA and has an $R^{2}$ of 0.994 and RMSE of $0.21 \mathrm{mg} / \mathrm{L}$. Note that for this analysis, each ANN was trained a 100 times to account for the randomness in training ANN caused by the random-start procedure of the training algorithm as well as to account for the limited size of the dataset used. The perfor- mance metrics are averaged over the 100 iterations.

Table 4. The Performance of ANN Modelling with Different Input Combinations

\begin{tabular}{llll}
\hline Combination No. & Input parameters & $R^{2}$ & RMSE \\
\hline 1 & EC, TP, T & 0.994 & 0.21 \\
2 & EC, T & 0.986 & 0.3 \\
3 & EC, TP & 0.925 & 0.68 \\
4 & T, TP & 0.941 & 0.61 \\
5 & EC & 0.884 & 0.83 \\
6 & T & 0.679 & 1.42 \\
7 & TP & 0.819 & 1.06 \\
\hline
\end{tabular}

Figure $5 \mathrm{~b}$ shows the trend plot of the predicted DO using the final ANN model. Note that the grey shaded area represents the results from the 100 repeated training results (effectively providing upper and lower bounds of the predictions at each point). The figure shows that the ANN model can follow the trend of the observed DO closely (as shown by the high performance metrics) and can capture the DO values within its bounds.

Note that in many cases, TP measurements cannot be conducted remotely or continuously (i.e., it requires a laboratory analysis rather than a sensor). Thus, an alternative approach may be to select the model that only uses EC and T to predict $\mathrm{DO}$, both of which are relatively simple to measure online and continuously. Even this approach, with one less input, provides a higher performance ANN model with an $R^{2}$ of 0.986 and RMSE of $0.3 \mathrm{mg} / \mathrm{L}$ (as shown in Table 4 above). It should be noted that by eliminating TP from the inputs, the performance of the models decreases slightly but generally is still high. Thus, by applying this IVS method, an accurate estimate of hypolimnetic DO can be achieved by only using upstream EC and T in the upstream river location as inputs to the model.

\subsection{Comparison of the Two DDMs}

For the MLR and the ANN models, the $R^{2}$ values were 0.857 and 0.994 , respectively, and the RMSE were $0.89 \mathrm{mg} / \mathrm{L}$ and $0.21 \mathrm{mg} / \mathrm{L}$, respectively. Thus, the ANN model demon- strates better performance using both performance criteria. Note that the MLR model does fail under one assumption, as identified above meaning that the resultant predictions are biased. A comparison of the predictions from both models are illustrated in Figure 6. The figure shows that the ANN model predictions fall closer to the 1:1 line than the MLR, indicating a better fit (and quantified using $R^{2}$, earlier). Also, note that the ANN model has fewer outliers, and generally predicts lower DO (a focus of this research) better than the MLR approach. The primary reason for this difference in performance is the ability of ANN to consider the non-linear and synergistic relationships between the input variables and DO, which the MLR approach cannot account for. The results of Table 2 to Table 4 reflect the sensitivity analysis of different parameters considering different DDMs. The results in Table 2 showed that there is not a significant linear relationship between $\mathrm{T}$ and hypolimnetic DO, meaning that the MLR is unable to account for the impacts of T on the hypolimnetic DO leading to lower model performance. This is confirmed looking at the results in Table 4 that show the ANN model performance increases substantially when $\mathrm{T}$ is added as an input variable (i.e. Combination No. 2 and 5), with the $R^{2}$ increasing from 0.884 to 0.986 . In other words, the non-linear relationship is captured by the ANN model but not the MLR model. Table 2 also demonstrates that EC has the highest linear correlation with hypolimnetic DO: using EC as the sole input to both models produce reasonable results with $R^{2}$ values of 0.814 and 0.884 for the MLR and ANN models, respectively. However, as discussed above, the ANN model performance increases to $R^{2}=0.986$ once T is also included as an input in the ANN (which was not selected as a candidate input for the MLR model). Similarly, TP has a high linear correlation with DO (as seen in Table 2); when comparing the MLR and ANN models that use both EC and TP as inputs, the ANN model performance is higher $\left(R^{2}=0.925\right.$ than the MLR model $\left(R^{2}=0.814\right)$. This demonstrates that the ANN model is better able to replicate the physio-chemical processes that govern DO concentration as compared to the ANN - important input variables such as T, TP and EC result in better performing ANN models, whereas the same inputs have lower performance in the MLR models. The ability of ANNs to model both the non-linear and synergistic effects of the inputs is the primary reason for this phenomenon.

In addition to comparing the model performance metrics for each method, five different hypothesis tests were conducted to examine the statistical significance of the difference between the two models and the observed data. Based on the results of these tests (as shown in Table 5), the null hypothesis has not been rejected and therefore, no statistically significant difference was found between the predict DO using each method, and between the predictions and the observations. This highlights that both models were able to capture the overall trends seen in the observation closely (i.e. no significant difference), and highlights that the models themselves were not very different between each other (though one underlying assumption for MLR was not met). However, as mentioned above the ANN model had better performance overall, and was better able to predict the low DO observations seen in the reservoir. 
Table 5. Comparing the Results of Simulation by ANN and MLR by Different Hypothesis Tests

\begin{tabular}{llll}
\hline Hypothesis test & $\begin{array}{l}\text { Comparing MLR results and } \\
\text { Observed DO }\end{array}$ & $\begin{array}{l}\text { Comparing ANN results and } \\
\text { Observed DO }\end{array}$ & $\begin{array}{l}\text { Comparing ANN results and MLR } \\
\text { results }\end{array}$ \\
\hline t-test & $\mathrm{h}^{*}=0$ & $\mathrm{~h}=0$ & $\mathrm{~h}=0$ \\
& $\mathrm{p}$-value $=0.95$ & $\mathrm{p}$-value $=0.94$ & $\mathrm{p}$-value $=0.99$ \\
Rank-sum test & $\mathrm{h}=0$ & $\mathrm{~h}=0$ & $\mathrm{~h}=0$ \\
& $\mathrm{p}$-value $=1$ & $\mathrm{p}$-value $=0.97$ & $\mathrm{p}$-value $=0.99$ \\
Signed-rank test & $\mathrm{h}=0$ & $\mathrm{~h}=0$ & $\mathrm{~h}=0$ \\
& $\mathrm{p}$-value $=0.38$ & $\mathrm{p}$-value $=1$ & $\mathrm{p}$-value $=0.38$ \\
KS-test & $\mathrm{h}=0$ & $\mathrm{~h}=0$ & $\mathrm{~h}=0$ \\
& $\mathrm{p}$-value $=0.83$ & $\mathrm{p}$-value $=1$ & $\mathrm{p}$-value $=0.83$ \\
F-test & $\mathrm{h}=0$ & $\mathrm{~h}=0$ & $\mathrm{~h}=0$ \\
& $\mathrm{p}$-valu $=0.87$ & $\mathrm{p}$-value $=0.91$ & $\mathrm{p}$-value $=0.96$ \\
\hline *h $=1$ indicates a rejection of the null hypothesis, and $\mathrm{h}=0$ indicates a failure to reject the null hypothesis at the 5\% significance level.
\end{tabular}

$* \mathrm{~h}=1$ indicates a rejection of the null hypothesis, and $\mathrm{h}=0$ indicates a failure to reject the null hypothesis at the $5 \%$ significance level.

Overall, as the results of the research demonstrate that the ANN model can better predict low hypolimnetic DO concentration in the study area. However, an important feature of the proposed approach is that the final configuration of the model uses input variables that are relatively easy and inexpensive to measure in-situ using a multi-parameter water quality sonde (i.e., these input variables do not require laboratory analysis). This suggests that by using the proposed approach, upstream water quality measurements can predict downstream DO in real-time, allowing water resource managers to take precautions to improve water quality as needed. In particular, this approach can help eliminate the need for a more comprehensive monitoring program to measure hypolimnetic DO (which is comparatively harder to sample).

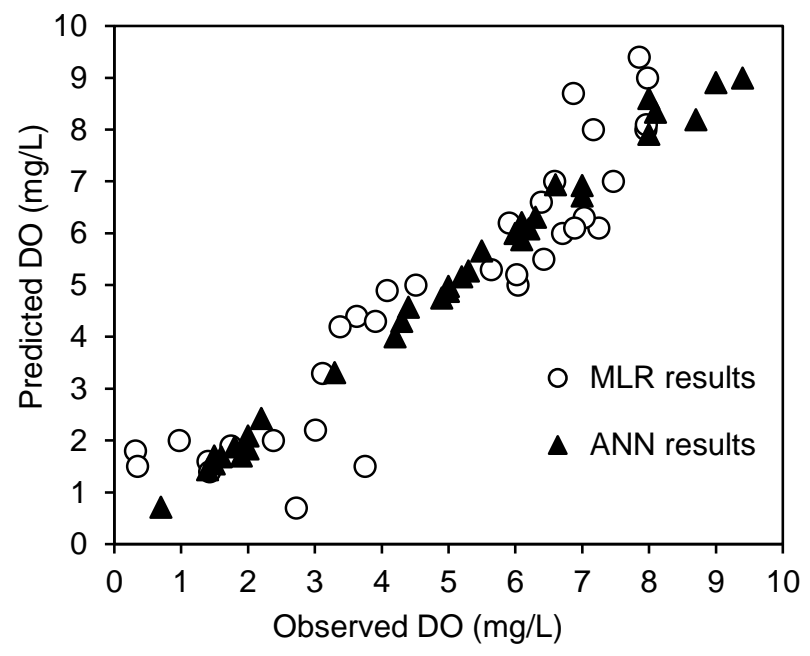

Figure 6. The results of the ANN and MLR models versus the observed data.

\section{Conclusions}

The level of hypolimnetic DO in large reservoirs has a key effect on the other water quality parameters such as phosphorus, ammonia and hydrogen sulphide and as a result, and it is considered an important parameter in water quality monitoring net- works for dams and reservoirs. Measurement of this indicator variable is not simple and thus, the use of numerical models to predict this variable under different conditions is extremely important. Thus, in this research, two DO models (MLR and ANN) were successfully developed for the Seymareh Reservoir, Iran, where low DO is a concern.

As part of the model calibration procedure, a systematic IVS method was implemented to determine the most effective input parameters for each modelling approach (a significant component of DDM design that is not widely researched). In this step, less important factors were eliminated from the analysis resulting in more efficient numerical models. The MLR approach used a correlation based IVS technique, whilst the ANN approach used an ANN-based IVS technique (the CNPSA method) that has not been compared to its linear counterparts in the literature.

The hypolimnetic DO in the Seymareh Reservoir were predicted using each method and the performances of the models were quantified using $R^{2}$ and RMSE. This analysis demonstrated that the ANN method performed better than the MLR method. The $R^{2}$ and RMSE for MLR model were 0.857 and $0.89 \mathrm{mg} / \mathrm{L}$ respectively, whilst for the ANN model the values were 0.994 and $0.21 \mathrm{mg} / \mathrm{L}$. Hypothesis tests were used to confirm that there was no significant difference between either of the model outputs and the observations, nor between the two types of models. However, the MLR approach failed to hold one underlying assumption, whereas the ANN model was particularly suited to predicting low DO. This comparison showed that considering the complexity of the natural processes that govern the water quality in reservoirs, the ANN model can provide more accurate forecasts of hypolimnetic DO than the MLR approach (which assumes linear relationships between the inputs and outputs).

In conclusion, the ANN model using CNPSA provided a more robust simulation of DO in the Reservoir. By using this method, the reservoir and dam operators or water resources managers can estimate the DO in the deeper layers of the reservoir by only using the measurements of online monitoring station on the upstream river. This research showed that the ANN approach is successful even with a relatively short dataset to train, validate and test the model which is another important 
achievement of this research. In addition, this research showed that IVS, particularly the CNPSA method, is an effective tool to select only the important input variables, reducing redundant information in the model, and hence, developing an efficient model with high performance. Future research should focus on further analysing the uncertainty in these types of DDMs, particularly, by considering the uncertainty in observed data, as well as comparing the results from DDMs to physically-based mod$1 \mathrm{~s}$, and using the developed models for scenario analysis including anthropogenic changes to the system and climate change.

Acknowledgements. The authors would like to thank: the Iranian Ministry of Energy for funding the monitoring program; and the Natural Sciences and Engineering Research Council of Canada; and York University for funding this research. We would also like to thank the feedbacks from the two anonymous reviewers whose comments helped improve the quality of this manuscript.

\section{References}

Abba, S.I., Sinan, J.H., and Abdullahi, J. (2017). River Water Modelling Prediction Using Multi-Linear Regression, Artificial Neural Network, and Adaptive Neuro-Fuzzy Inference System Techniques. Procedia Computer Science, 120, 75-82. https://doi.org/10.1016/ j.procs.2017.11.212.

Basant, N., Shikha, G., Amrita, M., and Kunwar, P.S. (2010). Linear and Nonlinear Modeling for Simultaneous Prediction of Dissolved Oxygen and Biochemical Oxygen Demand of the Surface Water - A Case Study. Chemometrics and Intelligent Laboratory Systems, 104(2), 172-180. https://doi.org/10.1016/j.chemolab.2010.08.005.

Bocaniov, S.A., Leon, L.F., Rao, Y.R., Schwab, D.J., and Scavia, D. (2017). Corrigendum to "simulating the effect of nutrient reduction on hypoxia in a large lake (Lake Erie, USA-Canada) with a threedimensional lake model" [(Journal of Great Lakes Research, 42 (2016) 1228-1240], Journal of Great Lakes Research, 43(4), 777. https://doi.org/10.1016/j.jglr.2017.07.006.

Brito, D., Ramos, T.B., Gonçalves, M.C., Morais, M., and Neves, R. (2018). Integrated Modelling for Water Quality Management in a Eutrophic Reservoir in South-Eastern Portugal, Environmental Earth Sciences 77(2), 1-20. https://doi.org/10.1007/s12665-017-72 21-5.

Burkholder, J.M., Mallin, M.A., and Jr Glasgow, H.B. (1999). Fish Kills, Bottom-Water Hypoxia, and the Toxic Pfiesteria Complex in the Neuse River and Estuary, Marine Ecology Progress Series, 179, 301-310. https://doi.org/10.3354/meps179301.

Chen, W.B. and Liu, W.C. (2015). Application of Multivariate Linear Regression and Neural Network Models for Simulating Water Quality in Reservoir, Journal of Taiwan Agricultural Engineering, 61(3). https://doi.org/10.1155/2015/521721.

Cheng, C.T., Kwokwing, C., Sun, Y.G., and Lin, J.Y. (2005). LongTerm Prediction of Discharges in Manwan Reservoir Using Artificial Neural Network Models, Lncs, 3498, 1040-1045.https://doi.org/ $10.1007 / 11427469165$.

Cooke, G. (2005). Restoration and Management of Lakes and Reservoirs, CRC press.

Dawson, C.W. and Wilby, R.L. (2001). Hydrological Modelling Using Artificial Neural Networks, Progress in Physical Geography, 25(1), 80-108. https://doi.org/10.1177/030913330102500104.

Duncan, A.P. (2014). The Analysis and Application of Artificial Neural Networks for Early Warning Systems in Hydrology and the Environment, Ph.D. Dissertation, College of Engineering, Mathematics and Physical Sciences, University of Exeter, Devon, UK.

Durbin, J., and Watson, G.S. (1951). Testing for Serial Correlation in
Least Squares Regression. II, Biometrika, 38(1/2), 159. https://doi. org/10.2307/2332325.

Ferraro, M.B. and Giordani, P. (2012). A Multiple Linear Regression Model for Imprecise Information, Metrika, 75(8), 1049-1068. https: //doi.org/10.1007/s00184-011-0367-3.

Hamilton, D.P. and Schladow, S.G. (1997). Prediction of Water Quality in Lakes and Reservoirs. Part I - Model Description, Ecological Modelling, 96(1-3), 91-110. https://doi.org/10.1016/S0304-3800(9 6)00062-2.

He, J.X., Chu, A., Ryan, C., Valeo, C., and Zaitlin, B. (2011). Abiotic Influences on Dissolved Oxygen in a Riverine Environment, Ecological Engineering, 37(11), 1804-1814. https://doi.org/10. 1016/j.ecoleng.2011.06.022.

Heddam, S. and Kisi, O. (2018). Modelling daily dissolved oxygen concentration using least square support vector machine, multivariate adaptive regression splines and M5 model tree, Journal of Hydrology, 559, 499-509. https://doi.org/10.1016/j.jhydrol.2018. 02.061 .

Jarque, C.M. and Anil, K.B. (1987). A Test for Normality of Observations and Regression Residuals. International Statistical Review / Revue Internationale de Statistique, 55(2), 163. https://doi.org/ $10.2307 / 1403192$

Bierlein, K.A., Rezvani, M., Socolofsky, S.A., Bryant, L.D., Wüest, A., and Little, J.C. (2017). Increased sediment oxygen flux in lakes and reservoirs: The impact of hypolimnetic oxygenation. Water Resources Research, 53(6), 4876-4890. https://doi.org/10.1002/ 2016WR019850.

Mosaad, K. and Elshemy, M. (2017). Data-Driven Modeling for Water Quality Prediction Case Study: The Drains System Associated with Manzala Lake, Egypt, Ain Shams Engineering Journal, 8(4), 549557. https://doi.org/10.1016/j.asej.2016.08.004.

Khan, U.T. and Valeo, C. (2016). Short-Term Peak Flow Rate Prediction and Flood Risk Assessment Using Fuzzy Linear Regression, Journal of Environmental Informatics, 28(2), 71-89. https://doi.org/ 10.3808/jei.201600345

Khan, U.T. and Valeo, C. (2016). Dissolved Oxygen Prediction Using a Possibility Theory Based Fuzzy Neural Network, Hydrology and Earth System Sciences, 20(6), 2267-2293. https://doi.org/10.5194/ hess-20-2267-2016

Khan, U.T. and Valeo, C. (2017). Comparing a Bayesian and Fuzzy Number Approach to Uncertainty Quantification in Short-Term Dissolved Oxygen Prediction, Journal of Environmental Informatics, 30(1), 1-16. https://doi.org/10.3808/jei.201700371.

Khan, U.T., He, J.X., and Valeo, C. (2018). River Flood Prediction Using Fuzzy Neural Networks: An Investigation on Automated Network Architecture, Water Science and Technology, 77(9), 107.

Khan, U.T. and Valeo, C. (2015). A New Fuzzy Linear Regression Approach for Dissolved Oxygen Prediction, Hydrological Sciences Journal, 60(6), 1096-1119. https://doi.org/10.1080/02626667.2014. 900558.

Khan, U.T. and Valeo, C. (2017). Optimising Fuzzy Neural Network Architecture for Dissolved Oxygen Prediction and Risk Analysis, Water, 9(6). https://doi.org/10.3390/w9060381.

Kisi, O., Akbari, N., Sanatipour, M., Hashemi, A., Teimourzadeh, K., and Shiri, J. (2013). Modeling of dissolved oxygen in river water using artificial intelligence techniques, Journal of Environmental Informatics, 22(2), 92-101. https://doi.org/10.3808/jei.201300248.

Kovdienko, N.A., Polishchuk, P.G., Muratov, E.N., Artemenko, A.G., Kuz'min, V.E., Gorb, L., Hill, F., and Leszczynski, J. (2010). Application of Random Forest and Multiple Linear Regression Techniques to QSPR Prediction of an Aqueous Solubility for Military Compounds, Molecular Informatics, 29(5), 394-406. https://doi.org/10.1002/minf.201000001.

Liu, W.C. and Chuan, E.C. (2014). Enhancing the Predicting Accuracy of the Water Stage Using a Physical-Based Model and an Artificial Neural Network-Genetic Algorithm in a River System, Water, 6(6), 
1642-1061. https://doi.org/10.3390/w6061642.

Lopez, C.B., Jewett, E.B., Dortch, Q., Walton, B.T., and Hudnell, H.K. (2008). Scientific assessment of freshwater harmful algal blooms, Interagency Working Group on Harmful Algal Blooms, Hypoxia, and Human Health of the Joint Subcommittee on Ocean Science and Technology, Washington, D.C., 1-78.

Newman, D. (1939). The Distribution of Range in Samples from a Normal Population, Expressed in Terms of an Independent Estimate of Standard Deviation, Biometrika, 31(1), 20-30. https://doi.org/10. 1093/biomet/31.1-2.20.

Kalpić D., Hlupić N., and Lovrić M. (2011). Student's t-Tests. In: Lovric M. (eds) International Encyclopedia of Statistical Science. Springer, Berlin, Heidelberg. https://doi.org/10.1007/978-3-64204898-2.

Quilty, J., Adamowski, J., Khalil, B., and Rathinasamy, M. (2016). Bootstrap rank-ordered conditional mutual information (broCMI): A nonlinear input variable selection method for water resources modeling, Water Resources Research, 52(3), 2299-2326. https://doi. org/10.1002/2015WR016959.

Rankovic, V., Radulovic, J., Radojevic, I., Ostojic, A., and Comic, L. (2010). Neural Network Modeling of Dissolved Oxygen in the Gruža Reservoir, Serbia, Ecological Modelling, 221(8), 1239-1244. https://doi.org/10.1016/j.ecolmodel.2009.12.023.

Rucinski, D.K., Beletsky, D., Depinto, J.V., Schwab, D.J., and Scavia, D. (2010). A Simple 1-Dimensional, Climate Based Dissolved Oxygen Model for the Central Basin of Lake Erie, Journal of Great Lakes Research, 36(3), 465-476. https://doi.org/10.1016/j.jglr.2010. 06.002.

Saghi, H., Karimi, L., and a. Javid, A.H. (2015). Investigation on Trophic State Index by Artificial Neural Networks (Case Study: Dez
Dam of Iran), Applied Water Science. https://doi.org/10.1007/s1320 1-014-0161-2

Singh, Kunwar P., Basant, A., Malik, A., and Jain, G. (2009). Artificial Neural Network Modeling of the River Water Quality-A Case Study, Ecological Modelling, 220(6), 888-895. https://doi.org/10.1016/j. ecolmodel.2009.01.004.

Kade, S., Kopf, R.K., Watts, R.J., and Howitt. J. (2014). Hypoxia, Blackwater and Fish Kills: Experimental Lethal Oxygen Thresholds in Juvenile Predatory Lowland River Fishes, PLoS ONE 9(4). https://doi.org/10.1371/journal.pone.0094524.

Snedecor, G.W. and Cochran, W.G. (1989). Statistical Methods, Eighth Edition. Iowa State University Press.

Solomatine, D.P. and Ostfeld, A. (2008). Data-Driven Modelling: Some Past Experiences and New Approaches, Journal of Hydroinformatics, 10(1), 3. https://doi.org/10.2166/hydro.2008.015.

Stefan, H.G. and Fang, X. (1994). Dissolved-Oxygen Model for Regional Lake Analysis, Ecological Modelling, 71(1-3), 37-68. https://doi.org/10.1016/0304-3800(94)90075-2.

Trolle, D., Spigel, B., Hamilton, D.P., Norton, N., Sutherland, D., Plew, D., and Mathew, G.A. (2014). Application of a Three-Dimensional Water Quality Model as a Decision Support Tool for the Management of Land-Use Changes in the Catchment of an Oligotrophic Lake, Environmental Management, 54(3), 479-93. https://doi.org/ 10.1007/s00267-014-0306-y.

Valeo, C. and He, J. (2009). Comparative Study of ANNs versus Parametric Methods in Rainfall Frequency Analysis, Journal of Hydrologic Engineering, 14(2), 172-184. https://doi.org/10.1061/(A SCE)1084-0699(2009)14:2(172).

Wilcoxon, F. (1945). Individual Comparisons by Ranking Methods, Biometrics Bulletin, 1(6), 80. https://doi.org/10.2307/3001968. 